\title{
Tradução e adaptação transcultural de instrumentos de avaliação em Fonoaudiologia para o português brasileiro: uma análise das diretrizes
}

\section{Translation and cross-cultural adaptation of Speech-Language Pathology assessment instruments to Brazilian Portuguese: guidelines analysis}

\author{
Nayara Ribeiro da Silva* \\ Leila Maria Gumushian Felipini**
}

Resumo: No Brasil, há escassez de instrumentos formais e objetivos comercialmente disponíveis em português e indicados para avaliação e diagnóstico na área de Fonoaudiologia. Uma forma que alguns pesquisadores têm encontrado para amenizar esse problema é traduzir instrumentos já disponíveis em outras línguas.

* Bacharel em Tradução pela Universidade do Sagrado Coração e mestranda em Fonoaudiologia pela Faculdade de Odontologia de Bauru - Universidade de São Paulo. E-mail: nayararibeiro28@hotmail.com.

** Doutora em Ciências pela Faculdade de Odontologia de Bauru. Docente da Universidade do Sagrado Coração. E-mail: leila.felipini79@gmail.com. 
Historicamente, a adaptação de instrumentos elaborados em outra cultura e/ou idioma se detinha à simples tradução do original ou à comparação literal dessa tradução com uma retrotradução. No entanto, há algum tempo pesquisadores de diferentes áreas temáticas vêm sugerindo que a avaliação semântica seja apenas um dos passos necessários ao processo de adaptação transcultural. Neste sentido, são propostos na literatura alguns conjuntos de instruções padronizadas e diretrizes específicas para a tradução e a adaptação transcultural de instrumentos. 0 objetivo deste estudo é apresentar as diretrizes recorrentes em estudos que executam a tradução e a adaptação transcultural de instrumentos. Para isso, foram selecionados 27 artigos científicos, indexados em bases de dados nacionais e internacionais, que descreviam esse processo em suas metodologias. As oito diretrizes verificadas - como as propostas por Guillemin, Bombardier e Beaton (1993) e pelo Scientific Advisory Committee of Medical Outcomes Trust (2002) - são consideradas bastante completas, o que reflete a grande preocupação por parte da maioria dos pesquisadores ao traduzir e adaptar instrumentos de avaliação em fonoaudiologia para uso no Brasil.

Palavras-chave: Tradução (Processo); Adaptação Transcultural; Diretrizes; Fonoaudiologia; Avaliação.

Abstract: In Brazil, there is a significant lack of formal and objective instruments that are commercially available in Portuguese for the assessment and diagnosis in the Speech-Language Pathology area. Some researchers have tried to ease this problem by translating instruments available in other languages. Historically, the adaptation of instruments developed in another culture and/or language was limited to the simple translation of the original or the literal comparison of this translation with a back translation. However, researchers from different thematic areas have been suggesting that semantic evaluation is only one of the necessary steps in the process of translation and cross-cultural adaptation. Hence, some sets of standardized instructions and specific guidelines for translation and cross-cultural adaptation of instruments are proposed in the literature. This study aims to present the recurrent guidelines in studies that perform the translation and cross-cultural adaptation of instruments. To do so, we selected 27 scientific articles indexed in national and international databases describing this process in their methodologies. The eight verified guidelines - as proposed by Guillemin, Bombardier, and Beaton (1993) and by the Scientific Advisory Committee of Medical Outcomes Trust (2002) - are considered quite comprehensive, reflecting the great concern of most researchers when translating and adapting Speech-Language Pathology assessment instruments for use in Brazil.

Keywords: Translation (Process); Cross-cultural Adaptation; Guidelines; SpeechLanguage Pathology; Assessment. 


\section{Introdução}

$\mathrm{Na}$ área fonoaudiológica, a avaliação é uma das ações mais frequentes na rotina de trabalho, e seu êxito é assegurado pelo alcance de informações que ajudem a levantar, confirmar ou negar uma hipótese diagnóstica (LINDAU ET AL. 2015). O objetivo da avaliação é elencar o conjunto de dificuldades e facilidades nos diferentes critérios avaliados, verificar e especificar os problemas e, ainda, obter dados para a tomada de decisões (SALVIA; YSSELDYKE 1991; Genaro et al. 2009; Pasquali 2010; Folha, 2010; Marchesan et Al. 2014).

A escassez de instrumentos formais e objetivos comercialmente disponíveis e indicados para avaliação e diagnóstico é significativa na área de Fonoaudiologia no Brasil (GIUSTI; BEFI-LOPES 2008; LINDAU ET AL. 2015). Os reais objetivos de um processo de avaliação clínica só podem ser atingidos quando os instrumentos e os procedimentos adequados são utilizados (GIUSTI; BEFILOPES 2008).

Além de refletir no diagnóstico, a falta de instrumentos formais e objetivos também reflete na definição das condutas terapêuticas e na elaboração dos planos de intervenção, chegando a comprometer a eficiência e a eficácia dos tratamentos oferecidos (ANDRADE 2004; ANDRADE 2005).

Uma forma que alguns pesquisadores têm encontrado para amenizar esse problema é traduzir instrumentos já disponíveis em outras línguas em vez de criar novos instrumentos (LINDAU; RosSI; GIACHETI 2014; ROCHA ET AL. 2014; NeVes et Al. 2014; Carvalho; Lúcio; Ávila 2015; Gonsalez; Almeida 2015; Zambon et al. 2016; Ayres et Al. 2016; Guedes-Granzotti et AL. 2016; Cruz et Al. 2017).

A tradução e a adaptação de instrumentos estrangeiros podem ser uma saída, porém os procedimentos adotados neste processo devem ser criteriosos e cuidadosos, uma vez que a tradução e a adaptação são tão importantes quanto a construção de um novo instrumento (GUILLEMIN; BOMBARDIER; BEATON 1993; Beaton et Al. 2000; Reichenheim; MORAes 2007). Além da preocupação e do cuidado metodológico que se deve ter nas pesquisas sobre a tradução e adaptação de instrumentos estrangeiros, também é importante que a aplicação e a interpretação do teste sejam criteriosas (GIUSTI; BEFI-LOPES 2008). 
O processo de tradução e adaptação transcultural de instrumentos para novas línguas/culturas apresenta diversas vantagens em relação ao desenvolvimento de um novo instrumento com a mesma finalidade (GUILLEMIN; Bombardier; Beaton 1993; Beaton et al. 2000; Pereira et al. 2013). Além de amenizar a carência de instrumentos disponíveis, tal procedimento pode contribuir para a realização de estudos transculturais, que podem trazer maiores esclarecimentos e compreensão sobre os quadros de distúrbios da comunicação e de suas especificidades nas diferentes línguas e culturas (GIUSTI; Befl-Lopes 2008). Além disso, esse procedimento pode permitir a comparação de diferentes populações e a troca de informações sem o viés das barreiras culturais e linguísticas (PEREIRA ET AL. 2013).

Historicamente, a adaptação de instrumentos elaborados em outra cultura e/ou idioma se detinha à simples tradução do original ou, excepcionalmente, à comparação literal dessa tradução com uma retrotradução. Há algum tempo, pesquisadores de diferentes áreas temáticas vêm sugerindo que a avaliação semântica constitua apenas um dos passos necessários ao processo de adaptação transcultural (BERKANOVICH 1980; PATRICK et al. 1985; Bucquet; Condon; Ritchie 1990; Bravo et al. 1991; Guillemin; Bombardier; Beaton 1993; Badia; Alonso 1995; Herdman; Fox-Rushby; Badia 1997; BEATON ET AL. 2000). Eles recomendam que o processo seja uma combinação entre a tradução literal de palavras e frases de um idioma ao outro, e um processo meticuloso de adaptação para o contexto cultural e estilo de vida da população-alvo da versão traduzida (GUILLEMIN; BoMBARDIER; BEATON 1993; Herdman; Fox-Rushby; Badia 1998; Beaton et al. 2000; Behling; LaW 2000; REICHENHEIM; MORAES 2007).

Partindo disso, alguns conjuntos de instruções padronizadas e diretrizes específicas para a tradução e a adaptação transcultural de instrumentos são propostos na literatura. Neste sentido, este estudo tem como objetivo apresentar as diretrizes recorrentes em estudos que executam a tradução e a adaptação transcultural de instrumentos. 


\section{Metodologia}

Para a realização deste estudo, foram selecionados 27 artigos científicos, indexados em bases de dados nacionais e internacionais, que descreviam em suas metodologias o processo de tradução e adaptação transcultural de algum instrumento de avaliação em fonoaudiologia, a fim de coletar dados referentes ao uso de diretrizes para a execução deste processo.

Para isso, utilizamos descritores combinados aos operadores booleanos da seguinte maneira: "Translation" $A N D$ "Adaptation" $O R$ "Cultural Adaptation" $O R$ "Cross-Cultural Adaptation" AND "Speech Language Pathology" nas bases de dados nacionais e internacionais Biblioteca Virtual em Saúde (BVS), Bireme (Lilacs, Ibecs e Adolec), PUBMED/MEDLINE, Scielo e Google Acadêmico.

Os critérios de exclusão estabelecidos para este estudo foram: (1) artigos repetidos por estarem publicados em mais de um idioma; (2) artigos repetidos localizados em mais de uma base de dados; (3) estudos realizados em outras línguas, como português europeu; (4) artigos que descreviam o processo de tradução e adaptação de instrumentos não pertencentes à área de Fonoaudiologia; (5) artigos que descreviam apenas o processo de validação de instrumentos.

\section{Resultados}

Para que não houvesse predição de resultados, foram selecionados aleatoriamente 27 artigos científicos que descreviam em suas metodologias 0 processo de tradução e adaptação transcultural de instrumentos de avaliação em fonoaudiologia, conforme demonstra a tabela 1.

Tabela 1: Dados referentes ao uso de diretrizes para a tradução e adaptação transcultural em cada artigo.

\begin{tabular}{l|l} 
Artigo & Diretriz
\end{tabular} 


\begin{tabular}{|c|c|c|c|}
\hline $\begin{array}{l}\text { Equivalência psicométrica da } \\
\text { versão brasileira do Test of Pragmatic } \\
\text { Language } 2 \text { - TOPL-2. }\end{array}$ & \multicolumn{2}{|c|}{ Beaton et al. (2000). } & \\
\hline $\begin{array}{l}\text { Planilha de triagem acústica da } \\
\text { sala de aula: tradução e adaptação } \\
\text { cultural para o Português Brasileiro. }\end{array}$ & $\begin{array}{l}\text { Guillemin, } \\
\text { Beaton (1993). }\end{array}$ & Bombardier & \\
\hline $\begin{array}{l}\text { Adaptação cultural do } \\
\text { questionário SADL (Satisfaction with } \\
\text { Amplification in Daily Life) para o } \\
\text { português brasileiro. }\end{array}$ & $\begin{array}{l}\text { Guillemin, } \\
\text { Beaton (1993). }\end{array}$ & Bombardier & e \\
\hline $\begin{array}{l}\text { Adaptação cultural do } \\
\text { questionário Speech, Spatial and } \\
\text { Qualities of Hearing Scale (SSQ) para o } \\
\text { Português Brasileiro. }\end{array}$ & $\begin{array}{l}\text { Guillemin, } \\
\text { Beaton (1993). }\end{array}$ & Bombardier & e \\
\hline $\begin{array}{l}\text { Versão brasileira do Dizziness } \\
\text { Handicap Inventory. }\end{array}$ & $\begin{array}{l}\text { Guillemin, } \\
\text { Beaton (1993). }\end{array}$ & Bombardier & \\
\hline $\begin{array}{l}\text { Early Listening Function (ELF): } \\
\text { adaptação para a língua portuguesa. }\end{array}$ & $\begin{array}{l}\text { Guillemin, } \\
\text { Beaton (1993). }\end{array}$ & Bombardier & \\
\hline $\begin{array}{l}\text { Tinnitus handicap inventory: } \\
\text { adaptação cultural para o Português } \\
\text { brasileiro. }\end{array}$ & $\begin{array}{l}\text { Guillemin, } \\
\text { Beaton (1993). }\end{array}$ & Bombardier & \\
\hline
\end{tabular}

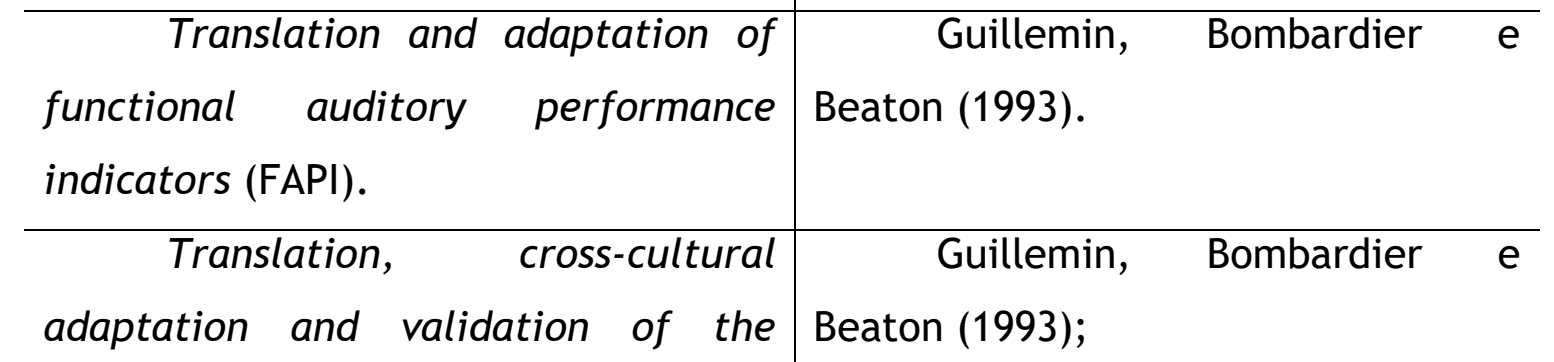
Portuguese version of the DYMUS Beaton et al. (2000). questionnaire for the assessment of dysphagia in multiple sclerosis.

\begin{tabular}{r|c} 
Versão brasileira do protocolo & Guillemin, Bombardier e \\
Overall Assessment of the Speaker's & Beaton (1993);
\end{tabular}
Experience of Stuttering - Adults Beaton et al. (2000). (OASES-A). 


\begin{tabular}{|c|c|}
\hline $\begin{array}{l}\text { Tradução e adaptação cultural } \\
\text { do Swallowing } \\
\text { Questionnaire para o português- } \\
\text { brasileiro. }\end{array}$ & $\begin{array}{l}\text { Guillemin, Bombardier e } \\
\text { Beaton (1993); } \\
\text { Beaton et al. (2000); } \\
\text { Peters e Passchier (2006). }\end{array}$ \\
\hline $\begin{array}{l}\text { Adaptação transcultural do } \\
\text { Preschool Language Assessment } \\
\text { Instrument: Segunda Edição. }\end{array}$ & $\begin{array}{l}\text { Guillemin, Bombardier e } \\
\text { Beaton (1993); } \\
\text { Herdman, Fox-Rushby e Badia } \\
\text { (1998); } \\
\text { Beaton et al. (2000); } \\
\text { Reichenheim e Moraes (2007); } \\
\text { Gjersing, Caplehorn e Clausen } \\
\text { (2010). }\end{array}$ \\
\hline $\begin{array}{l}\text { Adaptação do Dyslexia Early } \\
\text { Screening Test - Second Edition para o } \\
\text { Português Brasileiro: resultados } \\
\text { preliminares. }\end{array}$ & $\begin{array}{lllll}\text { Não relatou } & 0 & \text { uso } & \text { de } \\
\text { diretrizes. } & & & & \end{array}$ \\
\hline $\begin{array}{l}\text { Tradução e } \begin{array}{l}\text { adaptação } \\
\text { transcultural do Northwestern }\end{array} \\
\text { Dysphagia Patient Check Sheet para o } \\
\text { português brasileiro. }\end{array}$ & Peters e Passchier (2006). \\
\hline
\end{tabular}

Adaptação transcultural para o Reichenheim e Moraes (2007).

Português Brasileiro do Teste de Rastreamento Western Aphasia Battery - Revised: um estudo preliminar.

Tradução e adaptação cultural Reichenheim e Moraes (2007). brasileira do Detailed Assessment of Speed of Handwriting: equivalência conceitual e semântica.

Equivalência cultural da versão Scientific Advisory Committee brasileira do protocolo Evaluation of of Medical Outcomes Trust (2002). the Ability to Sing Easily.

Cross-cultural adaptation and Scientific Advisory Committee 
validation of the voice handicap index of Medical Outcomes Trust (2002). into Brazilian Portuguese.

Equivalência cultural da versão $\quad$ Scientific Advisory Committee Brasileira da Voice Symptom Scale - of Medical Outcomes Trust (2002). Voiss.

Equivalência cultural da versão Scientific Advisory Committee brasileira do Vocal Fatigue Index - VFI. of Medical Outcomes Trust (2002).

Qualidade de Vida em Voz na Scientific Advisory Committee População Pediátrica: validação da of Medical Outcomes Trust (2002). versão brasileira do Protocolo Qualidade de Vida em Voz Pediátrico.

Quality of life: validation of Scientific Advisory Committee the Brazilian version of the voice- of Medical Outcomes Trust (2002). related quality of life ( $V$-RQOL) measure.

Validação do Questionário de Scientific Advisory Committee Performance Vocal no Brasil. $\quad$ of Medical Outcomes Trust (2002).

Validação no Brasil de Scientific Advisory Committee
protocolos de auto-avaliação do of Medical Outcomes Trust (2002). impacto de uma disfonia.

Equivalência cultural da versão Scientific Advisory Committee brasileira do Eating Assessment Tool - of Medical Outcomes Trust (2002). EAT-10.

\begin{tabular}{|c|c|}
\hline $\begin{array}{l}\text { Tradução e avaliação preliminar } \\
\text { da versão em Português do } \\
\text { Questionário de Autoavaliação Vocal } \\
\text { para Transexuais de Homem para } \\
\text { Mulher. }\end{array}$ & $\begin{array}{l}\text { Wild et al. (2005); } \\
\text { Scientific Advisory Committee } \\
\text { of Medical Outcomes Trust (2002). }\end{array}$ \\
\hline $\begin{array}{lll}\text { Tradução e } & \text { adaptação } \\
\text { transcultural para o português } \\
\text { brasileiro do teste } \\
\text { Commildren's } \\
\text { Comication Checklist-2. }\end{array}$ & Wild et al. (2005). \\
\hline
\end{tabular}

Fonte: Elaborado pelo autor. 
Cinco autores optaram por seguir mais de uma referência de diretrizes para tradução e adaptação transcultural de instrumentos. Além das referências de diretrizes para tradução e adaptação transcultural de instrumentos, alguns autores referenciaram outros artigos em que o processo de tradução e adaptação transcultural de instrumentos foi executado - neste caso, apenas a referência de diretrizes foi utilizada na análise de dados para o resultado.

Um total de onze autores optou por seguir as diretrizes para a tradução e adaptação transcultural de instrumentos propostas por Guillemin, Bombardier e Beaton (1993), em "Cross-cultural adaptation of health-related quality of life measures: literature review and proposed guidelines". Esses autores explicam que a adaptação transcultural de um instrumento é composta por uma combinação de tradução literal de palavras e sentenças de um idioma para outro e uma adaptação com relação ao idioma, ao contexto cultural e ao estilo de vida do público-alvo. As diretrizes de Guillemin, Bombardier e Beaton (1993) são compostas por cinco etapas: (1) tradução; (2) retrotradução; (3) comitê de revisão; (4) pré-teste; e (5) possível adaptação dos pesos das pontuações para o contexto cultural.

Dez autores seguiram as diretrizes propostas pelo Scientific Advisory Committee (SAC) of Medical Outcomes Trust (2002), em "Assessing health status and quality of life instruments: attributes and review criteria". As etapas são cinco: (1) tradução; (2) conciliação; (3) retrotradução; (4) harmonização; e (5) teste piloto. O SAC recomenda critérios de padronização para desenvolvimento de protocolos de avaliação de qualidade de vida e validações em outros idiomas que não os originais. Segundo essas diretrizes, para que esses instrumentos possam ser utilizados em outras realidades culturais, eles devem ser traduzidos e adaptados de acordo com as regras internacionais e, em seguida, devem ter suas propriedades de medida demonstradas em um contexto cultural específico. Assim, o instrumento deve ser cuidadosamente traduzido e culturalmente adaptado, fugindo da simples tradução literal que exclui os contextos culturais e sociais. Ao final do processo, o protocolo traduzido e adaptado deve ser testado a fim de verificar 
sua adequação e validade para a população, o idioma e a cultura em questão, seguindo as regras internacionais de adaptação linguística e equivalência cultural, além de validade, reprodutibilidade, sensibilidade e confiabilidade.

Cinco desses artigos seguiram as diretrizes de Beaton et al. (2000) em "Guidelines for the Process of Cross-Cultural Adaptation of Self-Report Measures". As diretrizes propostas por Guillemin em 1993 foram aperfeiçoadas e reformuladas, e foram propostas seis etapas para a tradução e adaptação transcultural de instrumentos (BEATON ET AL. 2000): (1) tradução; (2) síntese das traduções; (3) retrotradução; (4) comitê de revisão; (5) préteste; e (6) submissão da documentação aos autores/comitê. De acordo com as diretrizes de 2000, existem recomendações a serem seguidas para se assegurar a compreensão total do conteúdo da tradução - como o uso de uma linguagem que possa ser compreendida por indivíduos de 10 a 12 anos de idade. Outras recomendações incluem a participação de profissionais qualificados, a repetição dos substantivos no lugar de pronomes e o uso de termos específicos em vez de termos mais gerais, além do uso de uma linguagem mais simples possível e a substituição da voz passiva pela voz ativa. É sugerido, também, que os autores (tradutores) evitem o uso de metáforas e coloquialismos, o modo subjetivo, advérbios e preposições se referindo a onde e quando, formas possessivas, palavras vagas e sentenças com dois verbos diferentes sugerindo ações diferentes.

Tanto as diretrizes propostas por Guillemin et al. (1993), quanto por Beaton et al. (2000), incluem orientações para se atingir uma equivalência semântica, idiomática, experiencial e conceitual entre o texto de partida e sua tradução. De acordo com Beaton et al. (2000), a equivalência semântica busca equacionar as dificuldades causadas por significados múltiplos, além de problemas gramaticais que podem surgir no processo de tradução. A equivalência idiomática se refere às expressões idiomáticas e coloquialismos. Nestes casos, expressões equivalentes devem ser usadas ou alguns itens devem ser substituídos. (BEATON ET AL. 2000). Para Guillemin et al. (1993), é mais provável que estas adequações sejam necessárias nas dimensões social e emocional. A equivalência experimental depende das situações evocadas no original se encaixarem no contexto da cultura-alvo. Beaton et al. (2000) 
definem este tipo de equivalência como sendo a tradução dos hábitos da vida diária de uma determinada cultura e explicam que uma tarefa específica pode não ser experimentada pelo povo de uma determinada cultura, como, por exemplo, o uso do garfo na alimentação que não é comum a todas as culturas. Já a equivalência conceitual se refere à validade do conceito explorado e às experiências vividas pelas pessoas na cultura-alvo, uma vez que algumas palavras podem apresentar equivalência semântica, mas não conceitual. Ou seja, o conceito de uma palavra varia de cultura para cultura. Beaton et al. (2000) citam como exemplo o conceito da palavra "família" que pode variar entre culturas, família nuclear ou extensa.

Três autores seguiram as diretrizes propostas por Reichenheim e Moraes (2007) em "Operacionalização de adaptação transcultural de instrumentos de aferição usados em epidemiologia". Essas diretrizes foram adaptadas para uso em estudos epidemiológicos a partir das diretrizes de Herdman, Fox-Rushby e Badia (1998) em "A model of equivalence in the cultural adaptation of HRQoL instruments: the universalist approach", elaboradas para o desenvolvimento de instrumentos de aferição sobre qualidade de vida. As diretrizes propõem um roteiro básico para o processo de tradução e adaptação transcultural de instrumentos, assumindo a postura "universalista", que abrange a apreciação de equivalência entre $o$ instrumento original e aquele a ser adaptado.

Segundo Herdman, Fox-Rushby e Badia (1998) e Reichenheim e Moraes (2007), a equivalência deve ser buscada de seis diferentes maneiras: (1) equivalência conceitual; (2) equivalência de item, que tem como finalidade explorar se os diferentes domínios abarcados pelo instrumento original na definição dos conceitos de interesse seriam relevantes e pertinentes ao novo contexto em que o instrumento será utilizado; (3) equivalência semântica, que envolve a transferência de sentido dos conceitos contidos no instrumento original para a versão traduzida; (4) equivalência operacional, que refere a uma comparação entre os aspectos de utilização de um instrumento nas populações-alvo e fonte, de modo que eficácia seja semelhante mesmo que os modus operandi não sejam os mesmos; (5) equivalência de mensuração, que se baseia na investigação das propriedades psicométricas do instrumento vertido; e (6) equivalência funcional, que não é elaborada, pois representa 
uma síntese das pregressas, como os próprios proponentes do modelo definem - é a etapa que enfatiza a importância de todas as etapas seguidas para se chegar à adaptação transcultural.

Dois dos autores optaram por seguir as diretrizes de Wild et al. (2005) em "Principles of good practice for the translation and cultural adaptation process for patient-reported outcomes (PRO) measures: report of the ISPOR Task Force for translation and cultural adaptation". Após identificar uma falta geral de consistência nos métodos atuais e nas diretrizes anteriormente publicadas, esse grupo de autores-pesquisadores percebeu a necessidade de desenvolver uma perspectiva holística que sintetizasse todo o espectro de métodos já publicados. Esse processo resultou no desenvolvimento do documento "Principles of good practice for the translation and cultural adaptation process for patient-reported outcomes (PRO) measures: report of the ISPOR Task Force for translation and cultural adaptation", um relatório sobre os métodos atuais $\mathrm{e}$ as diretrizes anteriormente publicadas, uma avaliação de seus pontos fortes e fracos e a proposta de um novo método. A nova proposta foi dividida em onze etapas, de acordo com o resultado obtido no estudo, sendo: (1) preparação, (2) tradução, (3) reconciliação, (4) retrotradução, (5) revisão da retrotradução, (6) harmonização, (7) revisão e aprovação pela autora principal do questionário original, (8) desdobramento cognitivo, (9) análise dos resultados do desdobramento cognitivo (10) revisão e (11) relatório final.

Dois dos artigos seguiram as diretrizes propostas por Peters e Passchier (2006) em "Translating instruments for cross-cultural studies in headache research". Trata-se de uma revisão que fornece um esboço das diretrizes e recomendações existentes que podem ser utilizadas para incentivar métodos de tradução rigorosos e de alta qualidade. Esses autores defendem que, além de seguir uma diretriz, é necessário que esse processo seja detalhadamente descrito quando publicado para que leitores e pesquisadores tenham acesso a essa informação. Para obter uma tradução de alta qualidade, Peters e Passchier (2006) consideram que as etapas a seguir devem ser incluídas no processo de tradução: (1) realizar traduções e retrotraduções; (2) seguir diretrizes para o processo de tradução e adaptação; (3) avaliar a qualidade e 
a equivalência da tradução; (4) realizar o teste piloto; e (5) avaliar as propriedades psicométricas da versão traduzida, como a consistência interna, a validade, a aplicabilidade e a confiabilidade.

Um dos autores optou por seguir as diretrizes propostas por Gjersing, Caplehorn e Clausen (2010) em “Cross-cultural adaptation of research instruments: language, setting, time and statistical considerations". Este artigo ilustrou o processo e os passos necessários para a adaptação transcultural de instrumentos de pesquisa, realizando o processo de tradução e adaptação transcultural de um instrumento como exemplo. Os autores enfatizam que os questionários nem sempre são traduzidos adequadamente antes de serem utilizados em novas configurações temporais, culturais ou linguísticas e por isso sugerem que o processo siga as seguintes etapas: (1) investigação de equivalência conceitual e de item; (2) tradução; (3) síntese das traduções; (4) retrotradução; (5) síntese das retrotraduções; (6) comitê de especialistas; (5) pré-teste do instrumento; (6) revisão do instrumento; (7) investigação da equivalência operacional; (8) teste do instrumento; (9) análise exploratória e de confirmação; e (10) versão final do instrumento.

Um dos autores não utilizou ou não relatou o uso de nenhuma diretriz para tradução e adaptação transcultural de instrumentos.

\section{Conclusão}

A prática de tradução e adaptação transcultural de instrumentos estrangeiros vem crescendo nos últimos anos, e, consequentemente, pesquisas sobre esta temática têm sido amplamente executadas, o que resultará, em um futuro próximo, na disponibilidade de um maior número de instrumentos de diagnóstico em fonoaudiologia.

Por serem consideradas bastante completas, as diretrizes verificadas demonstram a grande preocupação que a maioria dos pesquisadores vem tendo ao traduzir e adaptar instrumentos de avaliação em Fonoaudiologia para uso no Brasil. Não por menos, já que esses instrumentos terão grande 
impacto na prática clínica e na pesquisa e constituirão um passo fundamental para a identificação dos problemas mais frequentes na área fonoaudiológica e de seus fatores de risco, permitindo o melhor planejamento das avaliações, das intervenções e dos tratamentos oferecidos.

\section{Referências bibliográficas}

AARONSON, N. et al. Assessing health status and quality of life instruments: attributes and review criteria. Qual Life Res, v. 11, n. 3, Oxford, may. 2002, pp. 193-205.

ANDRADE, C. R. F.; JUSTE, F. Proposta de análise de performance e de evolução em crianças com gagueira desenvolvimental. Rev CEFAC, v. 7, n. 2, São Paulo, abr./jun. 2005, pp. 158-170. Disponível em: <http://www.redalyc.org/pdf/1693/Resumenes/Resumo_1693205020 03_5.pdf> Acesso em: 18 jul. 2017.

ANDRADE, C. R. F. A fonoaudiologia baseada em evidências. Einstein, v. 2, n. 1, 2004, pp. 59-60.

AYRES, A. ET AL. Tradução e adaptação cultural do swallowing disturbance questionnaire para o português-brasileiro. Rev CEFAC, v. 18, n. 4, São Paulo, ago. 2016, pp. 828-834. Disponível em: <http://www.scielo.br/pdf/rcefac/v18n4/1982-0216-rcefac-18-0400828.pdf>. Acesso em: 16 jul. 2017.

BADIA, X.; Alonso, J. Re-scaling the Spanish version of the Sickness Impact Profile: an opportunity for the assessment of cross-cultural equivalence. J Clin Epidemiol, v. 48, n. 7, Oxford, jul. 1995, pp. 949957.

BEATON, D. E ET AL. Guidelines for the process of cross-cultural adaptation of self-report measures. Spine, v. 25, n. 24, Philadelphia, dec. 2000, pp. 3186-3191.

Behlau, M.; Alves, S. L. M.; Oliveira, G. Cross-cultural adaptation and validation of the voice handicap index into Brazilian Portuguese. $J$ Voice, v. 25, n. 3, New York, may. 2011, pp. 354-359.

Behlau, M. et AL. Validação no Brasil de protocolos de auto-avaliação do impacto de uma disfonia. Pró-Fono, v. 21, n. 4, Barueri, dez. 2009, 
pp. 326-332. Disponível em:

<http://www.scielo.br/pdf/pfono/v21n4/11.pdf>. Acesso em: 17 jul. 2017.

BEHLING, O.; LAW, K. S. Translating questionnaires and other research instruments: problems and solutions. Thousand Oaks: Sage; 2000.

BERKANOVICH, E. The effect of inadequate language translation of Hispanics' responses to health surveys. Am J Public Health, v. 70, n. 12, New York, dec. 1980, pp. 1273-1276.

BRAGATTO, E. L. ET AL. Versão brasileira do protocolo Overall Assessment of the Speaker's Experience of Stuttering - Adults (OASES-A). J Soc Bras Fonoaudiol, v. 24, n. 2, São Paulo, 2012, pp. 145-151. Disponível em: <http://www.scielo.br/pdf/jsbf/v24n2/pt_10.pdf>. Acesso em: 16 jul. 2017.

BRAvo, M. ET AL. A cross-cultural adaptation of a psychiatric epidemiologic instrument: the diagnostic interview schedule's adaptation in Puerto Rico. Cult Med Psychiatry, v. 15, n. 1, Dordrecht, mar.1991, pp. 1-18.

BucqueT, D.; CONDON, S.; RITCHIE, K. The French version of the Nottingham Health Profile. A comparison of items weights with those of the source version. Soc Sci Med, v. 30, n. 7, Oxford, 1990, pp. 829-835.

Cardoso, M. H.; Henderson, S.; Capellini, S. A. Tradução e adaptação cultural brasileira do Detailed Assessment of Speed of Handwriting: equivalência conceitual e semântica. Audiol, Commun Res, v. 19, n. 4, São Paulo, dez. 2014, pp. 321-326. Disponível em: <http://www.scielo.br/pdf/acr/v19n4/pt_2317-6431-acr-231764312014000400001447.pdf>. Acesso em: 18 jul. 2017.

Carvalho, C. A. F.; Lúcio, P. S.; Ávila, C. R. B. Psychometric equivalence of the Brazilian version of the Test of Pragmatic Language 2 - TOPL-2. CoDAS, v. 27, n. 4, São Paulo, aug. 2015, pp. 344-349. Disponível em: <http://www.scielo.br/pdf/codas/v27n4/pt_2317-1782-codas-27-0400344.pdf>. Acesso em 20 jul. 2017.

CAstro, A. S. O. ET AL. Versão brasileira do Dizziness Handicap Inventory. PróFono, v. 19, n. 1, Barueri, abr. 2007, pp. 97-104. Disponível em: <http://www.scielo.br/pdf/pfono/v19n1/10.pdf>. Acesso em: 20 jul. 2017.

COSTA, V. B. S. ET AL. Tradução e adaptação transcultural para o português brasileiro do teste Children's Communication Checklist-2. CoDAS, v. 25, n. 2, São Paulo, 2013, pp. 115-119. Disponível em: TradTerm, São Paulo, v.32, dezembro/2018, p. 32-51 
<http://www.scielo.br/pdf/codas/v25n2/a05v25n2.pdf>. Acesso em: 19 jul. 2017.

CRUZ, A. D. ET AL. Planilha de triagem acústica da sala de aula: tradução e adaptação cultural para o Português Brasileiro. Audiol, Commun Res., v. 22, São Paulo, 2017. Disponível em:

<http://www.scielo.br/pdf/acr/v22/2317-6431-acr-2317-6431-20161766.pdf>. Acesso em: 19 jul. 2017.

FERREIRA, K. ET AL. Translation and adaptation of functional auditory performance indicators (FAPI). J Appl Oral Sci, v. 19, n.6, Bauru, dec. 2011, pp. 586-598. Disponível em: <http://www.scielo.br/pdf/jaos/v19n6/a08v19n6.pdf> Acesso em: 18 jul. 2017.

FERREIRA, P. E. A. ET AL. Tinnitus handicap inventory: adaptação cultural para o Português brasileiro. Pró-Fono, v. 17, n. 3, Barueri, dez. 2005, pp. 303-310. Disponível em:

<http://www.scielo.br/pdf/pfono/v17n3/v17n3a03.pdf>. Acesso em 19 jul. 2017.

FolHA, G. A. Ampliação das escalas numéricas do Protocolo de Avaliação Miofuncional Orofacial (AMIOFE), validação e confiabilidade. 2010. Dissertação (Mestrado em Morfofisiologia de Estruturas Faciais) Faculdade de Medicina de Ribeirão Preto, Universidade de São Paulo, Ribeirão Preto, 2010.

GaSPARINI, G; BehlaU, M. Quality of life: validation of the Brazilian version of the voice-related quality of life (V-RQOL) measure. J Voice, v. 23, n. 1, New York, jan. 2009, pp. 76-81.

GeNARO, K. F. ET AL. Avaliação miofuncional orofacial: protocolo MBGR. Rev CEFAC, v. 11, n. 2, São Paulo, jun. 2009, pp. 237-255. Disponível em: <http://www.scielo.br/pdf/rcefac/v11n2/v11n2a09.pdf>. Acesso em: 20 jul. 2017.

GIUSTI, E.; Befl-LoPES, D. M. Tradução e adaptação transcultural de instrumentos estrangeiros para o Português Brasileiro (PB). Pró-Fono, v. 20, n. 3, Barueri, set. 2008, pp. 207-210. Disponível em: <http://www.scielo.br/pdf/pfono/v20n3/12.pdf>. Acesso em: 20 jul. 2017.

GJeRsing, L.; Caplehorn, J.; Clausen, T. Cross-cultural adaptation of research instruments: language, setting, time and statistical considerations. BMC Med Res Methodol, v. 10, n. 1, London, fev. 2010. 
Gonçalves, M. I. R.; Remailı, C. B.; Behlau, M. Equivalência cultural da versão brasileira do Eating Assessment Tool - EAT-10. CoDAS, v. 25, n. 6, São Paulo, 2013, pp. 601-604. Disponível em:

<http://www.scielo.br/pdf/codas/2013nahead/aop_292_13.pdf>. Acesso em: 29 jul. 2017.

Gonsalez, E. C. M.; Almeida, K. Adaptação cultural do questionário Speech, Spatial and Qualities of Hearing Scale (SSQ) para o Português Brasileiro. Audiol, Commun Res, v. 20, n. 3, São Paulo, set. 2015, pp. 215-224. Disponível em: <http://www.scielo.br/pdf/acr/v20n3/23176431-acr-20-3-0215.pdf>. Acesso em: 20 jul. 2017.

Guedes-GranzotTI, R. B. ET AL. Adaptação transcultural do Communication Function Classification System para indivíduos com paralisia cerebral. Rev CEFAC, v. 18, n. 4, São Paulo, ago. 2016, pp. 1020-1028. Disponível em: <http://www.scielo.br/pdf/rcefac/v18n4/1982-0216rcefac-18-04-01020.pdf>. Acesso em: 20 jul. 2017.

Guillemin, F.; Bombardier, C.; Beaton, D. Cross-cultural adaptation of healthrelated quality of life measures: literature review and proposed guidelines. J Clin Epidemiol, v. 46, n. 12, Oxford, dec. 1993, pp. 1417-1432.

Herdman. M.; Fox-Rushby, J.; Badia, X. "Equivalence" and the translation and adaptation of health-related quality of life questionnaires. Qual Life Res, v. 6, n. 3, Oxford, apr. 1997, pp. 237-247.

Herdman, M.; Fox-Rushby, J.; BAdiA, X. A model of equivalence in the cultural adaptation of HRQol Instruments: The universalist approach. Qual Life Res, v. 7, n. 4, Oxford, may. 1998, pp. 323-335.

LINDAU, T. A. et al. Instrumentos sistemáticos e formais de avaliação da linguagem de pré-escolares no brasil: uma revisão de literatura. Rev CEFAC, v. 17, n. 2, São Paulo, abr. 2015, pp. 656-662. Disponível em: <http://www.scielo.br/pdf/rcefac/v17n2/1982-0216-rcefac-17-0200656.pdf>. Acesso em 19 jul. 2017.

Lindau, T. A.; Rossi, N. F.; GiAChetI, C. M. Preschool Language Assessment Instrument, segunda edição, em crianças falantes do Português Brasileiro. CoDAS, v. 26, n. 4, São Paulo, jul. 2014, pp. 328-330. Disponível em: <http://www.scielo.br/pdf/codas/v26n4/pt_23171782-codas-26-04-00328.pdf>. Acesso em 18 jul. 2017.

MAGALHÃES, J. H. V. ET AL. Tradução e adaptação transcultural do Northwestern Dysphagia Patient Check Sheet para o português brasileiro. CoDAS, v. 25, n. 4, São Paulo, 2013, pp. 369-374. Disponível em: 
<http://www.scielo.br/pdf/codas/v25n4/12.pdf >. Acesso em: 18 jul. 2017.

Marchesan, I. Q.; Berretin-Felix, G.; Genaro, K. F. MBGR - Protocolo de Avaliação em Motricidade Orofacial com Escores. In: TESSITORE, A.; Marchesan, I. Q.; Justino, H.; Berretin-Felix, G. (org.) Práticas clínicas em motricidade orofacial. Pinhais: Editora Melo, 2014. pp. 97-116.

MATTA, T. R. G.; BefI-Lopes, D. M. Adaptação do Dyslexia Early Screening Test Second Edition para o Português Brasileiro: resultados preliminares. CoDAS, v. 27, n. 3, São Paulo, jun. 2015, pp. 301-303. Disponível em: <http://www.scielo.br/pdf/codas/v27n3/pt_2317-1782-codas-27-0300301.pdf>. Acesso em: 21 jul. 2017.

MondelLI, M. F. C. G.; MAgalhães, F. F.; LaURIS, J. R. P. Adaptação cultural do questionário SADL (Satisfaction with Amplification in Daily Life) para o português brasileiro. Braz J Otorhinolaryngol, v. 77, n. 5, São Paulo, oct. 2011, pp. 563-572. Disponível em:

<http://www.scielo.br/pdf/bjorl/v77n5/pt_v77n5a05.pdf>. Acesso em: 21 jul. 2017.

MORETI, F. ET AL. Equivalência cultural da versão Brasileira da Voice Symptom Scale: VoiSS. J Soc Bras Fonoaudiol, n. 4, v. 23, São Paulo, dez. 2011, pp. 398-400. Disponível em:

<http://www.scielo.br/pdf/jsbf/v23n4/v23n4a18.pdf> Acesso em: 21 jul. 2017.

Neves, M. B. ET AL. Cross-cultural adaptation of the Western Aphasia Battery Revised screening test to Brazilian Portuguese: a preliminary study. CoDAS, n. 1, v. 26, São Paulo, fev. 2014, pp. 38-45. Disponível em: <http://www.scielo.br/pdf/codas/v26n1/pt_2317-1782-codas-26-0100038.pdf>. Acesso em: 20 jul. 2017.

Oshima, M. et AL. Early Listening Function (ELF): adaptação para a língua portuguesa. Rev Soc Bras Fonoaudiol, v. 15, n. 2, São Paulo, 2010, pp. 191-196. Disponível em:

<http://www.scielo.br/pdf/rsbf/v15n2/08.pdf>. Acesso em: 20 jul. 2017.

Pasqual, L. Taxonomia dos instrumentos psicológicos. In: Pasqual, L. Instrumentação psicológica: fundamentos e práticas. Rio de Janeiro: Artmed, 2010. pp. 48-55.

PATRICK, D. L. ET AL. A cross-cultural comparison of health status values. Am J Public Health Res, v. 75, n. 12, Newark, dec. 1985, pp. 1402-1407. 
Paulinelli, B. R.; Gama, A. C. C.; Behlau, M. Validação do Questionário de Performance Vocal no Brasil. J Soc Bras Fonoaudiol, v. 17, n. 1, São Paulo, mar. 2012, pp. 85-91. Disponível em: <http://www.scielo.br/pdf/rsbf/v17n1/a16v17n1.pdf>. Acesso em: 22 jul. 2017.

PereiRA, L. M. ET AL. Translation, cross-cultural adaptation and analysis of the psychometric properties of the lower extremity functional scale (LEFS): LEFS- BRAZIL. Braz J Phys Ther, v. 17, n. 3, São Carlos, jun. 2013, pp. 272-280. Disponível em: <http://www.scielo.br/pdf/rbfis/v17n3/1413-3555-rbfis-17-030272.pdf> Acesso em: 22 jul. 2017.

Peters, M.; PASSChier, J. Translating Instruments for Cross-Cultural Studies in Headache Research. Headache, v. 46, n. 1, St. Louis, jan, 2006, pp. 82-91.

REICHENHEIM, M. E.; MORAES, C. L. Operationalizing the cross-cultural adaptation of epidemiological measurement instruments. Rev Saúde Pública, v. 41, n. 4, São Paulo, aug. 2007, pp. 665-673.

Ribeiro, L. L.; PaUla, K. M. P.; Behlau, M. Qualidade de Vida em Voz na População Pediátrica: validação da versão brasileira do Protocolo Qualidade de Vida em Voz Pediátrico. CoDAS, v. 26, n. 1, São Paulo, fev. 2014, pp. 87-95. Disponível em: <http://www.scielo.br/pdf/codas/v26n1/pt_2317-1782-codas-26-0100087.pdf>. Acesso em 22 jul. 2017.

RochA, B. R. ET AL. Cross-cultural adaptation of the Brazilian version of the protocol Evaluation of the Ability to Sing Easily. CoDAS, n. 6, v. 26, São Paulo, dez. 2014, pp. 535-539. Disponível em: <http://www.scielo.br/pdf/codas/v26n6/pt_2317-1782-codas-26-0600535.pdf>. Acesso em: 20 jul. 2017.

SALES, D. S. ET AL. Translation, cross-cultural adaptation and validation of the Portuguese version of the DYMUS questionnaire for the assessment of dysphagia in multiple sclerosis. Springerplus, v. 2, Switzerland, jul. 2013, pp. 332.

SALVIA, J.; YSSELDYKE, J. E. Avaliação: em educação especial e corretiva. 4 ed. São Paulo: Manole, 1991.

SANTOS, H. H. A. N. M. ET AL. Tradução e avaliação preliminar da versão em Português do Questionário de Autoavaliação Vocal para Transexuais de Homem para Mulher. CoDAS, v. 27, n. 1, São Paulo, feb. 2015, pp. 89- 
96. Disponível em <http://www.scielo.br/pdf/codas/v27n1/pt_23171782-codas-27-01-00089.pdf>. Acesso em: 22 jul. 2017.

WAng, W.; LeE, H.; Fetzer, S. Challenges and strategies of instrument translation. West J Nursing Res, v. 28, n. 3, Beverly Hills, apr. 2006, pp. 310-321.

WILD, D. ET AL. Principles of good practice for the translation and cultural adaptation process for patient-reported outcomes (PRO) measures: report of the ISPOR Task Force for translation and cultural adaptation. Value Health, v. 8, n. 2, Malden, mar/apr. 2005, pp. 94-104.

ZAMBON, F. ET AL. Equivalência cultural da versão brasileira do Vocal Fatigue Index - VFI. CoDAS, v. 29, n. 2, São Paulo, 2017. Disponível em: <http://www.scielo.br/pdf/codas/v29n2/2317-1782-codas-2317178220172015261 .pdf>. Acesso em: 18 jul. 2017.

Recebido em: 05/10/2018

Aceito em: 11/12/2018 Publicado em dezembro de 2018 